Original paper

\title{
Whole exome sequencing analysis for mutations in isolated type III biliary atresia patients
}

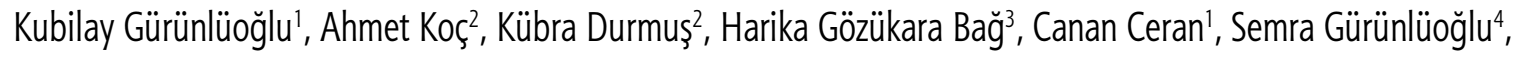 \\ Turan Yıldız', Mehmet Güls ${ }^{5}$ Mehmet Demircan' \\ 'Department of Pediatric Surgery, Faculty of Medicine, İnönü University, Malatya, Turkey \\ 2Department of Medical Genetics, Faculty of Medicine, Inönü University, Malatya, Turkey \\ ${ }^{3}$ Department of Biostatistics and Medical Informatics, Faculty of Medicine, Inönü University, Malatya, Turkey \\ ${ }^{4}$ Pathology Laboratory, Education and Research Hospital, Malatya, Turkey \\ ${ }^{5}$ Department of Histology and Embryology, Faculty of Medicine, Inönü University, Malatya, Turkey
}

\begin{abstract}
Aim of the study: Biliary atresia is an idiopathic, destructive disease that affects both extrahepatic and intrahepatic bile ducts with severe inflammation and manifests as progressive jaundice within the first few months of life. In this study, we aimed to investigate the significance of genetic mutations in the onset of biliary atresia disease.

Material and methods: With the approval of the ethics committee and parental consent, blood was taken from patients to obtain their DNA, and the study commenced. In this prospective study, we examined the DNA of 10 patients with no disease other than biliary atresia, and an exome sequence analysis was performed with the new-generation DNA sequencing method. The genetic structure of biliary atresia disease was examined by statistical analysis of the mutations, which were determined according to the reference DNA sequencing.

Results: In the exome sequence analysis, the number of mutations detected among the patients changed significantly; the lowest number was 12,591 , and the maximum was 19,863 . By examining these mutations, we identified the mutated genes that were common to all patients.

Conclusions: In this study, the highest mutation rates were detected in the PRIM2 and MAP2K3 genes. These genes have not previously been associated with biliary atresia.
\end{abstract}

Key words: biliary atresia, DNA, exome sequencing analysis, mutation, genetic.

Address for correspondence:

Dr. Kubilay Gürünlüoğlu, Department of Pediatric Surgery, Faculty of Medicine, İnönü University, Camurlu street, Postal code: 44210, Malatya, Turkey, e-mail: kgurunluoglu@hotmail.com

\section{Introduction}

Biliary atresia $(\mathrm{BA})$ is a rare disease that emerges in the first months of life, increasing at the level of direct bilirubin. In BA disease, there is an obstruction in the extrahepatic and intrahepatic bile ducts, but its etiology has not been fully illuminated. Inflammation and destructive damage to the liver may occur in BA disease and can result in final stage liver failure if left untreated $[1,2]$.

The incidence of BA ranges from 1 in 10,000 to 1 in 16,700 [1]. Three main types have been defined. Type I has atresia at the site of the common bile duct, type II has atresia at the site of the hepatic duct, and type III has atresia at the site of the porta hepatis. The most common is type III (84.1\%) [1].

In infants, BA is suspected in cases with acholic stools and the appearance of jaundice in the first weeks after birth. To diagnose BA, hematological, serological, and biochemical evaluation, ultrasonographic evaluation, hepatobiliary scintigraphy, and percutaneous liver biopsy should be performed. The last procedure to be performed for diagnosis is intraoperative cholangiography with surgical exploration [1]. 
Hepatic portoenterostomy (the Kasai procedure) is the standard surgical treatment for BA [2].

Although in the etiology of BA, genetic, infectious, and immuno-mediated factors are suspected of playing important roles, they have not been proven [1].

Important studies have been conducted on the effect of genetic factors in the etiology of BA, and it has been claimed that certain genes may be associated [3]. The aim of this study is to contribute to understanding the etiology of this disease using next-generation exome sequence analysis in BA patients to identify common mutations among them.

\section{Material and methods}

This single-center (Turgut Özal Medical Center Training and Research Hospital, Department of Pediatric Surgery) study was approved by the Clinical Research Ethics Committee of the Faculty of Medicine of İnönü University Malatya/Turkey [No. 2018/19]. The study protocol conformed to the provisions of the Declaration of Helsinki. Informed consent was obtained from all patients' parents.

During the study period, 26 patients were eligible for inclusion; 10 patients were excluded due to the exclusion criteria, 4 patients declined to take part, and 2 patients could not be included for some reasons (e.g., not enough DNA is available, parents' consent not feasible).

In this prospective study, $10 \mathrm{BA}$ patients between 55 and 70 days of age who met the inclusion and exclusion criteria were treated, which included Kasai portoenterostomy surgery. The İnönü University Malatya

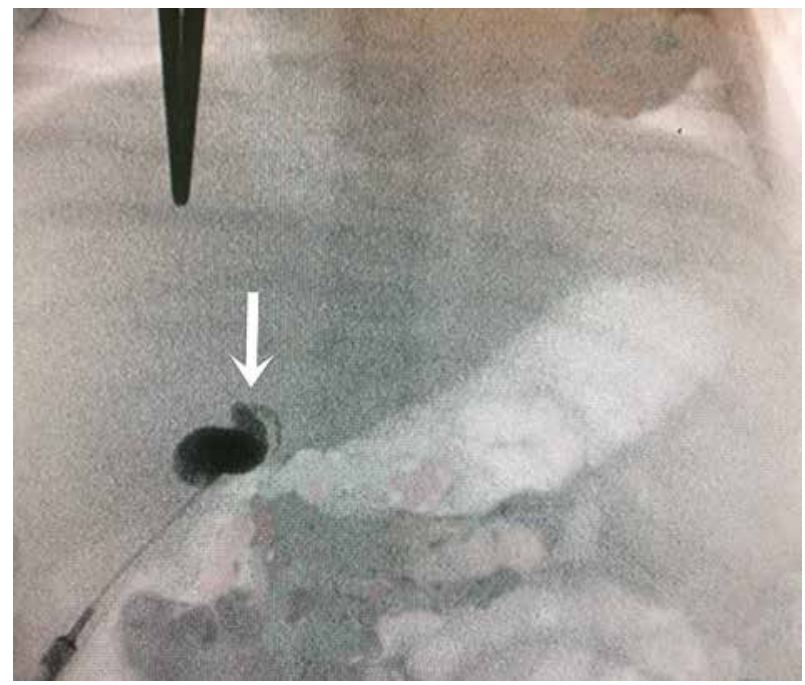

Fig. 1. Intraoperative cholangiographic image of a 57-day-old female patient with isolated type III biliary atresia. The radiopaque fluid passes from the gallbladder to neither the liver nor the intestines (white arrow)
Clinical Research Ethics Board (2018/19) granted approval for the study to begin. Due to suspected BA, intra-operative cholangiogram (Fig. 1) was performed, and 10 patients diagnosed with $\mathrm{BA}$ received the necessary approval from their parents for DNA analysis. All patients underwent Kasai portoenterostomy surgery after diagnosis (Fig. 2). Demographics of patients are shown in Table 1. Tissue for liver biopsy was collected from all patients during the operation, and BA findings were confirmed by histopathologic examination of biopsied tissue.

Patients who had been diagnosed with intra-operative BA found to be type III, did not have major additional anomalies, and were 55-70 days old at the time of the operation were included. From patients who met these criteria, a blood sample for genetic examination was collected immediately after the surgery.

Patients who had a diagnosis of non-intraoperative BA, whose subtype was other than type III, who were not 55-70 days old at operation time, whose blood sample could not be collected immediately after the operation, or who had an additional major anomaly were excluded.

\section{Collecting blood samples from patients}

For genetic analysis, 2-ml blood samples were drawn intravenously from the upper right or left extremity of each patient, with the Seldinger technique, into 2-ml blood vacuum tubes (Vacutainer, BDPlymouth, UK) containing K2 ethylenediaminetetraacetic acid. The blood samples were frozen at $-80^{\circ} \mathrm{C}$ until testing.

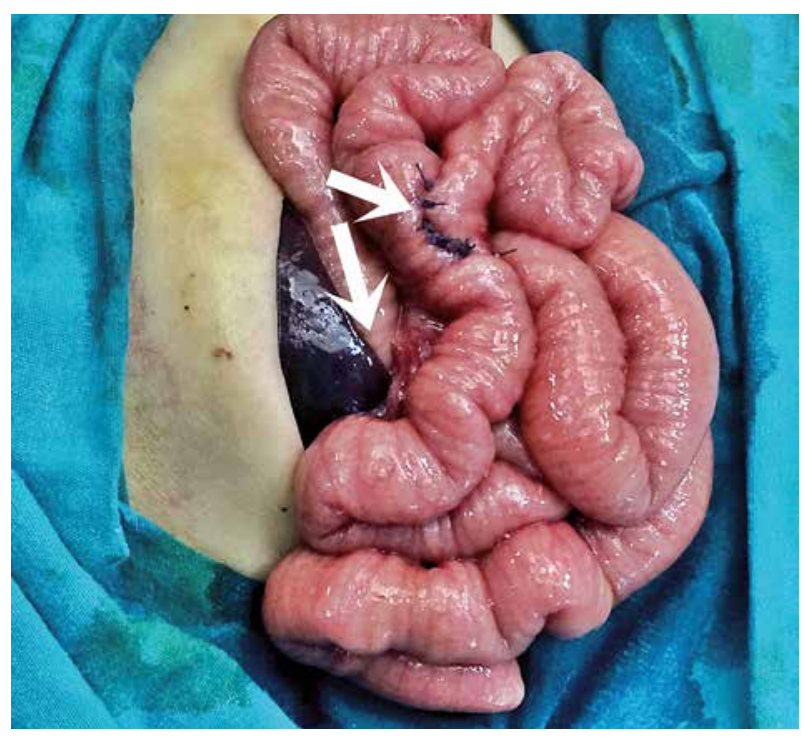

Fig. 2. Completed Kasai hepatic portoenterostomy operation in an isolated type III biliary atresia patient (white arrows - Roux-en-Y anastomosis areas) 
Table 1. Demographic information of the patients and number of genetic mutations detected in each patient

\begin{tabular}{cccccc}
\hline Gender & Age of operation day (years) & Serum TB $(\mathbf{m g} / \mathrm{dl})$ & Serum DB $(\mathbf{m g} / \mathrm{dl})$ & Another anomaly & Number of mutations \\
\hline F & 63 & 12.70 & 10.50 & Non & 12591 \\
\hline F & 57 & 8.21 & 6.22 & Non & 18128 \\
\hline F & 46 & 20.18 & 18.62 & Non & 17302 \\
\hline M & 62 & 7.65 & 5.72 & Non & 17641 \\
\hline M & 86 & 9.04 & 8.50 & Non & 16229 \\
\hline F & 61 & 9.80 & 10.1 & Non & 18909 \\
\hline F & 68 & 15.5 & 16.8 & Non & 19691 \\
\hline M & 70 & 7.8 & 8.8 & Non & 12688 \\
\hline M & 72 & 11.3 & 6.4 & Non & 13242 \\
\hline F & 51 & 12.9 & 16.7 & Non & 19863 \\
\hline
\end{tabular}

\section{DNA isolation from blood samples}

In the molecular genetics laboratory of the Inonu University School of Medicine, genomic DNA was isolated from the blood samples using a GeneJET whole-blood genomic DNA purification mini kit. DNA concentration was determined using a nanodrop spectrophotometer (DS-11 Fx+, Denovix). The DNA materials obtained from patients were then sent to Genoks Genetic Diseases Diagnostic Center, Cankaya, Ankara, Turkey.

\section{Exome sequence analysis}

Exome sequencing enables the enrichment of exons within the coding regions of the human genome and analysis of mutations caused by potential variations with high-yield sequences [4]. The main advantage of the GenoXome clinical whole-exome alignment panel is that it provides a simple and cost-effective approach to assist clinical diagnosis in identifying variants responsible for genetic disorders through advanced analysis and reporting. The methods used for screening more than 180,000 exons over 22,000 genes and the research on genetic mutations that can lead to complex or Mendelian diseases with genetic variation are described briefly below [4].

\section{Step 1: Genomic DNA isolation and quality-quantity determination}

Peripheral blood samples were taken from the patients, and genomic DNA isolation was performed using a kit as mentioned above. DNA concentrations and degradation conditions were examined according to the criteria for starting the sequencing of samples. The patients' DNA obtained was sent to Genoks for later processing. The following procedures were carried out by the Genoks company, and the clinical reports and VCF files were returned to us [4].

\section{Step 2: Library preparation}

The quality-controlled genomic DNA sample was randomly divided into trailers using the sonication technique, and the trailer sizes generally selected were those between 150 BC and 250 BC. Nucleobase Adenine was added to the 3 ' end of each chain by repairing the ends of the DNA fragments. Adapters and barcodes were then connected to both ends of the fragments. DNA fragments were amplified with ligation-mediated polymerase chain reaction (LM-PCR) and then purified to remove those not connected. Hybridization was performed with a capture kit with a $59 \mathrm{MB}$ target zone for enrichment. Non-hybrid trailers were then washed and cleaned. The captured products were transformed into a circular chain in a circularization stage and amplified to form DNA nanoballs (DNB) on the same chain [4].

\section{Step 3: Sequencing stages}

Each DNB created was placed on the spots of the flow cell on the BGISEQ-500 sequencing platform. The sequencing was carried out on the flow cell. As in the classical sequencing method, the reaction solution containing modified dNTPs stained with different dyes was loaded into the flow cell. The BGI Seq 500 devices use combinatorial Probe-Anchor Synthesis (Cpas) technology to allow the bases to read over each nanoball. Sequencing operation includes primary binding, loading the sequence solution into the flow cell, connecting the first after the primary, and removal of unconnected bases in the environment. The of the flow cell photo of consisted of the respective stages. Sequences were made at a depth of 100X sequencing [4]. 


\section{Step 4: Bioinformatics analysis}

A series of file cycles and bioinformatics analyses were performed because data obtained from exome sequencing are raw data and to list variations [4]. To put it simply;

1. FASTQ files sent from BGI were sent as CLEAN DATA.

2. Clean data: The Burrows-Wheeler Aligner (BWA V0.7.15) was used to align with the reference genome hg19.

3. The resulting SAM file was sorted by coordinates with Picard tools, and duplicate readings were marked.

4. Sequences of small insertions and deletions (indels) were re-localized with GATK, and the BAM file was created.

5. Base Quality Score Recalibration calculated the quality score of each nucleobase.

6. Using the Target BED file, HaplotypeCaller GATK (v3.7), and the SNP and indel variant calling files were created.

7. The SNP and indels were filtered out and converted to a single file.

8. The merged and filtered VCF file was recorded.

9. Clinical reports were prepared.

\section{Statistical method}

In type III BA patients, the mutation incidence was predicted to be $80 \%$. To estimate this ratio with 0.10 deviation at a $95 \%$ confidence interval $(\alpha=0.05)$, the required minimum sample size was calculated as 6 .

\section{Results}

Table 1 shows the results of the new-generation exome sequence analysis of DNA samples from $10 \mathrm{pa}$ tients diagnosed with $\mathrm{BA}$ and the mutation numbers detected in each patient.

When these results were evaluated, 12,591 mutations were found in the patient with the fewest mutations, and 19,863 mutations were found in the patient with the highest number. All patients' mutations were examined, and the same mutations were found in some. Mutations in certain genes were found in the case of SNP (single nucleotide polymorphism, single nucleotide change). These mutations, which are the same in all patients, are shown in Table 2.

\section{Clinical evaluation of findings}

Our findings demonstrated that genetic mutations might play an essential role in the etiology of BA. Since no other anomaly or disease was present in our patients, perhaps BA occurs when all the mutations that were repeated in all our patients exist together. BA may be a pathology caused by the interaction of a single mutation in a single gene or many mutations in many genes. In this study, the chromosomes, genes, and mutations specifically responsible for BA were not revealed. Given the possibility of polymorphism, which is normally seen in humans, the majority of the mutations we encountered in patients are changes that have nothing to do with BA. Interestingly, two genes with multiple mutations were detected in all patients. Of these, 68 mutations belonging to the PRIM2 gene and 78 belonging to the MAK2K3 gene were found in all patients. This level of consistency (seen in all patients) suggests that there may be a link between the aforementioned genes and BA. The PRIM2 gene is involved in synthesizing RNA primaries used for the synthesis of Okazaki fragments during DNA synthesis. According to the Online Mendelian Inheritance in Man (OMIM) database, this gene has no connection to previously defined pathologies. The other gene, MAP2K3, encodes a protein kinase enzyme that is mitogenic, and this gene has also never been linked previously to BA.

\section{Discussion}

Many theories have been proposed to illuminate BA's etiology, and many studies have been conducted. Landing suggested one of these theories, which has come to the fore in recent years. The theory suggested that a viral infection in the bile ducts, over time, turned into an autoimmune disease structure, leading to the development of BA [5].

Morecki et al. claimed in their study that reovirus type III could play a role in BA's etiology [6].

In their study, Rauschenfels et al. examined liver biopsy samples of $74 \mathrm{BA}$ patients, and identified viral DNA/ RNA in 30 patients. They found that 21 of these viral DNA/RNAs belonged to the reovirus. They concluded that viral infections do not play an important role in BA etiology. According to their findings, viral infections may have been acquired after the onset of BA disease. They found that the inflammatory picture of the liver that presents when viral infections caused the liver infection is very similar to the inflammation detected in BA [7].

On the other hand, Nakashima et al. experimentally found that reovirus-type-II infection in newborn mice triggers an autoimmune inflammation induced by T-helper lymphocytes, which they claimed are seen in the bile ducts in BA [8].

We examined the results of preoperative routine laboratory examinations of the 10 patients included in our study and found 8 with PCR results of cytomegalovirus (CMV) [+]. Although we do not claim that 
Table 2. In 10 patients, commonly identified, mutated chromosomes, genes and SNPs

\begin{tabular}{|c|c|c|}
\hline Chromosome & Gene & $\begin{array}{l}\text { Number of } \\
\text { SNPs common } \\
\text { to } 10 \text { patients }\end{array}$ \\
\hline \multirow[t]{4}{*}{ Chr1 } & NBPF19, NBPF20, NBPF8, NBPF9 & 2 \\
\hline & NOC2L & 1 \\
\hline & S100PBP & 1 \\
\hline & DDX20 & 1 \\
\hline \multirow[t]{2}{*}{$\mathrm{Chr} 2$} & NT5DC4 & 1 \\
\hline & STK11IP & 1 \\
\hline \multirow[t]{2}{*}{ CHR3 } & ITPR1 & 1 \\
\hline & FNDC3B & 1 \\
\hline CHR4 & OTOP1 & 1 \\
\hline \multirow[t]{3}{*}{ CHR5 } & CATSPER3 & 1 \\
\hline & GEMIN5 & 1 \\
\hline & $\mathrm{PCDH} 1$ & 1 \\
\hline \multirow[t]{2}{*}{ CHR6 } & PRIM2 & 63 \\
\hline & SLC22A16 & 1 \\
\hline \multirow[t]{3}{*}{ Chr7 } & KMT2C & 4 \\
\hline & PRSS1 & 8 \\
\hline & INTS1 & 1 \\
\hline Chr8 & PCMTD1 & 1 \\
\hline \multirow[t]{4}{*}{ Chr9 } & MIR1299 & 4 \\
\hline & MIR4477B, LINC00537, MIR4477A & 7 \\
\hline & $A B C A 2$ & 1 \\
\hline & GALT & 1 \\
\hline Chr10 & GPRIN2 & 5 \\
\hline \multirow[t]{3}{*}{ Chr11 } & ATM & 1 \\
\hline & TENM4 & 1 \\
\hline & EIF4G2 & 1 \\
\hline
\end{tabular}

\begin{tabular}{|c|c|c|}
\hline Chromosome & Gene & $\begin{array}{l}\text { Number of } \\
\text { SNPs common } \\
\text { to } 10 \text { patients }\end{array}$ \\
\hline \multirow[t]{2}{*}{ Chr12 } & KRT4 & 1 \\
\hline & OTOGL & 1 \\
\hline Chr13 & NUFIP1 & 1 \\
\hline \multirow[t]{2}{*}{ Chr15 } & $F A H$ & 1 \\
\hline & GOLGA6L2 & 1 \\
\hline \multirow[t]{2}{*}{ Chr16 } & USP7 & 1 \\
\hline & ITFG1 & 1 \\
\hline \multirow[t]{6}{*}{ Chr17 } & MAP2K3 & 78 \\
\hline & KCNJ12, KCNJ18 & 4 \\
\hline & CCDC144NL & 3 \\
\hline & USPG & 2 \\
\hline & MPP2 & 1 \\
\hline & FTSJ3 & 1 \\
\hline Chr19 & SIGLEC5 & 1 \\
\hline \multirow[t]{2}{*}{ Chr2O } & TMEM189-UBE2V1, UBE2V1 & 1 \\
\hline & AURKA & 1 \\
\hline \multirow[t]{3}{*}{ Chr21 } & BAGE, BAGE2, BAGE3, BAGE4, BAGE5 & 4 \\
\hline & $B A G E$ & 3 \\
\hline & BAGE2, BAGE3, BAGE4, BAGE5 & 2 \\
\hline \multirow[t]{2}{*}{ Chr22 } & $\pi C 38$ & 1 \\
\hline & CASTOR1 & 1 \\
\hline ChrX & COL4A5 & 1 \\
\hline ChrY & $C D 24$ & 1 \\
\hline
\end{tabular}

a CMV-BA relationship exists, studies by Lakshminarayanan et al. highlighted their findings of CMV infection in BA patients [9].

Many studies have claimed that an autoimmune response in BA etiology, in which viral infection plays an essential role in its origin, can be significant [10-13].

Regarding the etiology of BA, it has been claimed that inflammation that occurs in the bile ducts during the intrauterine period or after birth and resulting in fibrosis may play a role. It is not clear how this inflammation develops $[1,3,13]$. It has been claimed that the triggering factor (viral infection, autoimmune response) may be a genetic mutation that was able to find a suitable environment $[1,3,13]$.

It was also reported by Lachaux et al. that genetic factors may play a role in BA's etiology. They reported two siblings diagnosed with familial extrahepatic BA,

which was thought to demonstrate autosomal recessive heredity [14].

Hartley et al. suggested that inflammation, which plays a vital role in the emergence of $\mathrm{BA}$, develops into $\mathrm{BA}$ if it is present in patients with a genetic predisposition. In these patients, affected genes that altered the existing inflammatory response were claimed to cause different inflammatory pathways in the affected liver that could lead to development and fibrosis. They reported that these affected genes were the hepcidin antimicrobial peptide gene, CD14 endotoxin receptor gene, ICAM1 gene, CFC1 gene, and the macrophage migration inhibitor factor gene [15].

The glypican-1 gene is located on the 2 q 37.3 chromosome. It was asserted that a single gene deletion in this area caused a defect in the development of the biliary tree despite there being no change in liver development [16]. 
Other genes that may be associated with a disorder in the development of bile ducts are CFTR, JAG1, ZIC3, CFC1, INV, MIF, VEGF, and IFN- $\gamma$ [17-22].

Cheng et al. claimed that 5-single nucleotide polymorphism (5-SNP) consisting of the 10q25.1 chromosome led to the appearance of $\mathrm{BA}$, causing the expression of the ADD3 genome to decrease [23]. The ADD3 genome specifically encodes the construction of adducin- $\gamma$, which provides adhesion among liver epithelial cells [24].

In studies to date, the $A D D 3$ and $G P C 1$ genes are the most potent genetic factors thought to play a role in BA etiology [16-23].

Rajagopalan et al. did a DNA sequence analysis in 101 isolated BA patients. They found mutations in the REV1 gene located on the second chromosome and in the STIP1 gene on the eleventh chromosome. $R E V 1$ codes a protein that is in charge of DNA repair. STIP1 is a gene that helps the protein transfer response in heat shock response. Rajagopalan et al. claimed that these mutations were due to exposure to toxic environmental factors [25].

In our study, we found no mutations in the genes mentioned above. However, among the mutations we identified were many SNPs in two genes (Table 2). One of these is the PRIM2 gene [26]. The PRIM2 gene is one of two subunits of DNA primase. DNA primase is a critical enzyme that catalyzes the synthesis of oligoribonucleotide during DNA replication [27].

There is no study supporting the relationship between the PRIM2 gene and BA, and only Dong et al. speculated that PRIM2 may be responsible for increasing the proliferation of inflammatory cells in posttraumatic sepsis [28].

The MAP2K3 gene is the other gene we found in our study in which a considerable number of SNPs occurred in all our BA patients. Mitogen-activated protein kinase-3 (MAP2K3) is a member of the dual-specificity kinase group [29], and may have been linked to tumor progression and invasion [29], but there is no study of a relationship between the MAP2K3 gene and $B A$. It has been claimed that only mice that are experimentally exposed to drinking water recurrently may have deregulation in the expression of the MAP2K3 gene in the liver [30].

In this study, whose aim was to demonstrate the role of genetic mutations in the etiology of BA, we acknowledge that there are certain limiting factors. Although our number of patients is statistically sufficient for power analysis, we recommend that studies involving more patients be carried out. If we had conducted a similar exome sequence analysis on our patients' parents, we would have had additional information about the type of heredity patterns of some mutations. Although all of our patients' parents are healthy, such mutations may be present in healthy people. However, due to our project's limited budget, we were unable to perform parental exome sequencing.

In summary, in this study we used a next-generation DNA sequencing approach to analyze exomes of 10 BA patients. We found many mutations, some of which were common to all patients. The PRIM2 and $M A P 2 K 3$ genes contained the highest number of mutual variations/mutations in all patients, which was a remarkable finding. We speculate that there is a pathway that renders an environment suitable for BA onset. We suspect that this pathway starts the BA process by being activated when mutations occur in many genes at the same time.

\section{Funding}

This project has been financed by Inonu University Scientific Research Projects Coordination Unit by TSA2018-1296 project code.

\section{Disclosure}

The authors declare no conflict of interest.

\section{References}

1. Altman RP, Buchmiller TL. The jaundiced infant: biliary atresia. In: Grosfeld JL, O’Neill JA, Fonkalsrud EW, Coran AG (Eds.). Textbook of Pediatric Surgery. $6^{\text {th }}$ ed. Vol. 2, Chapter 103. Philadelphia 2006: 1603-1619.

2. de Carvalho NMN, Torres SM, Cavalcante JCB, et al. Hepatoportoenterostomy surgery technique. J Pediatr Surg 2019; 54: 1715-1718.

3. Mezina A, Karpen SJ. Genetic contributors and modifiers of biliary atresia. Dig Dis 2015; 33: 408-414.

4. Xu Y, Lin Z, Tang C, et al. A new massively paralel nanoball sequencing platform for whole exome research. BMC Bioinformatics 2019; 20: 153.

5. Landing $\mathrm{BH}$. Considerations of the pathogenesis of neonatal hepatitis, biliary atresia and cholodekal cyst: the concept of infantile obstructive cholongiopathy. Prog Pediatr Surg 1974; 6: 113-139.

6. Morecki R, Glaser JH, Cho S, et al. Biliary atresia and reovirus type 3 infection. N Eng J Med 1982; 307: 481-484.

7. Rauschenfels S, Krassmann M, Al-Masri AN, et al. Incidence of hepatotropic viruses in biliary atresia. Eur J Pediatr 2009; 168: 469-476.

8. Nakashima T, Hayashi T, Tomoeda S, et al. Reovirus type-2triggered autoimmune cholangitis in extrahepatic bile ducts of weanling DBA/1J mice. Pediatr Res 2014; 75: 29-37.

9. Lakshminarayanan B, Davenport M. Biliary atresia: a comprehensive review. J Autoimmun 2016; 73: 1-9.

10. Mack CL, Sokol RJ. Unraveling the pathogenesis and etiology of biliary atresia. Pediatr Res 2005; 57: 87-97. 
11. Nakamura K, Tanoue A. Etiology of biliary atresia as a developmental anomaly: recent advances. J Hepatobiliary Pancreat Sci 2013; 20: 459-464.

12. Zhao D, Long XD, Xia Q. Recent advances in etiology of biliary atresia. Clin Pediatr 2015; 54: 723-731.

13. Petersen C, Davenport M. Aetiology of biliary atresia: what is actually known? Orphanet J Rare Dis 2013; 8: 128.

14. Lachaux A, Descos B, Plauchu H, et al. Familial extrahepatic biliary atresia. J Pediatr Gastroenterol Nutr 1988; 7: 280-283.

15. Hartley JL, Davenport M, Kelly DA. Biliary atresia. Lancet 2009; 374: 1704-1713.

16. Cui S, Leyva-Vega M, Tsai EA, et al. Evidence from human and zebrafish that GPC1 is a biliary atresia susceptibility gene. Gastroenterology 2013; 144: 1107-1115.

17. Davit-Spraul A, Baussan C, Hermeziu BB, et al. CFC1 gene involvement in biliary atresia with polisplenia syndrome. J Pediatr Gastroenterol Nutr 2008; 46: 111-112.

18. Arikan C, Berdeli A, Ozgenç F, et al. Positive ossociation of macrophage migration inhibitory factor gene-173G/C polymorphism with biliary atresia. J Pediatr Gastroenterol Nutr 2006; 42: 77-82.

19. Lee HC, Chang TTY, Yeung CY, et al. Association of interferon-gamma gene polymorphisims in Taiwanese children with biliary atresia. J Clin Immunol 2010; 30: 68-73.

20. Koshaka T, Yuan ZR, Guo SX, et al. The significance of human jagged 1 mutations detected in severe cases of extrahepatic biliary atresia. Hepatology 2002; 36: 904-912.

21. Uemura M, Ozawa A, Nagata T, et al. Sox17 haploinsufficiency results in perinatal biliary atresia and hepatitis in C57BL/6 backround mice. Development 2013; 140: 639-648.

22. Shimadera S, Iwai N, Deguchi E, et al. The inv mouse as an experimental model of biliary atresia. J Pediatr Surg 2007; 42: $1555-1560$

23. Cheng G, Tang CS, Wong EH, et al. Common genetic variants regulating $\mathrm{ADD} 3$ gene expression alter biliary atresia risk. J Hepatol 2013; 59: 1285-1291.

24. Citterio L, Tizzoni L, Catalano M, et al. Expression analysis human adducing gene family and evidence of ADD2 beta4 multiple splicing variants. Biochem Biophys Res Commun 2003; 309: 359-367.

25. Rajagopalan R, Tsai EA, Grochowski CM, et al. Exome sequencing in individuals with isolated biliary atresia. Sci Rep 2020; 10: 2709.

26. Chung J, Tsai S, James AH, et al. Lack of genomic imprinting of DNA primase, polypeptide 2 (PRIM2) in human term placenta and white blood cells. Epigenetics 2012; 7: 429-431.

27. Shiratori A, Okumura K, Nagomi M, et al. Assignment of the 49-kDa (PRIM1) and 58-kDa (PRIM2A and PRIM2B) subunits genes of the human DNA primase to chromosome bands 1q44 and 6p11.1-p12. Genomics 1995; 28: 350-353.

28. Dong L, Li H, Zhang S, Su L. Identification of genes related to consecutive trauma-induced sepsis via gene expression profiling analysis. Medicine (Baltimore) 2018; 97: e0362.

29. Gurtner A, Starace G, Norelli G, et al. Mutant p53-induced up-regulation of mitogen-activated protein kinase 3 contributes to gain of function. J Biol Chem 2010; 285: 14160-14169.

30. Wu B, Zhang Y, Zhao D, et al. Gene expression profiles in liver of mouse after chronic exposure drinking water. J Appl Toxicol 2009; 29: 569-577. 